\title{
Successful percutaneous stenting of coarctation of the aorta in Turner syndrome - a case report and literature review
}

\author{
Daniela Toma ${ }^{1,2}$, Dorottya-Anna Miklosi', Anca Sglimbea', Laszlo Hadadi',2, Marian Pop ${ }^{1,2}$, \\ Andreea Cerghit-Paler ${ }^{1,2}$, Amalia Fagarasan ${ }^{1,2}$, Rodica Toganel ${ }^{1,2}$, Liliana Gozar ${ }^{1,2}$ \\ ${ }^{1}$ Emergency Institute of Cardiovascular Diseases and Transplantation, Tg. Mures, Romania \\ 2 "George Emil Palade" University of Medicine, Pharmacy, Science and Technology, Tg. Mures, Romania
}

\begin{abstract}
Turner syndrome (TS) is the most common chromosomal abnormality affecting females and cardiac abnormalities have been described in up to $50 \%$ of patients. Although coarctation represents one of the most frequent cardiac malformation, treatment options in these patients represent an area of debate, due to associated aortopathy and risk for aortic dissection. In addition to the contradictory data found in the literature, regarding the safety profile and utility of stenting of coarctation of the aorta in TS patients, we present the case of a patient of pediatric age, who successfully underwent the procedure, being free from periprocedural and short-term complications. Beside the presentation itself, we aimed to review and summarize the data available in the literature regarding this topic. As a conclusion, we emphasise the role of minimally invasive interventional therapy and wish to underline the need of further, larger scale studies and guidelines in this patient group. Given the related aortopathy, all preventive measures should be undertaken to avoid aortic dissection during stent implantation in this vulnerable population. Although the evolution of our patient was favorable, data found in the literature is somewhat contradictory and a close follow-up is indicated to help evaluate the risk of long-term complications.
\end{abstract}

Keywords: Turner syndrome, coarctation, stenting, interventional therapy, pediatric

\section{INTRODUCTION}

Turner syndrome (TS) is the most common chromosomal abnormality affecting females, as it has a prevalence of $1 / 2.500$ in female newborns [1,2]. It is due to the complete or partial absence of the second $\mathrm{X}$ chromosome and may present clinical features such as: short stature, webbing of the neck, sexual infantilism, proeminent ears, epicanthal folds, broad chest, premature ovarian failure and lymphedema [2,3]. Associated malformations are common, thus making multiple organ dysfunction possible [4]. Up to $50 \%$ of TS patients are described to have congenital cardiac abnormalities, most frequently a bicuspid aortic valve, dilatation of the ascending aorta and coarctation of the aorta [1-3,5].

Although coarctation of the aorta is one of the most frequently encountered cardiac malformation in these patients, experience in different treatment protocols (surgical vs. percutaneous) is somewhat contradictory. The aim of this paper is to present the experience of our center with a TS patient of pediatric age, who successfully underwent and benefited from percutaneous stenting of an associated cardi- 
ac malformation: coarctation of the aorta. Subsequently, as data in the literature regarding the safety and utility of stenting in Turner syndrome patients are scarce and conflicting, we would also like to provide an overview of the data currently available. A comprehensive search was conducted, using PubMed database and the search engine Google Scholar.

\section{CASE PRESENTATION}

First of all we would like to present the case of a patient, reffered to our center at the age of 13. She was firstly diagnosed with TS, having associated endocrinopathies: Hashimoto thyroiditis with latent hypothyroiditis, and congenital dislocation of the hip, for which she benefited from surgical correction. Her first cardiologic evaluation performed at age 12 revealed coarctation of the aorta and arterial hypertension. Associated anti-hypertensive medication was initiated, with beta-blocker and angiotensin converting enzyme inhibitor, and she was reffered to our higher ranking institution for evaluation.

Upon clinical evaluation the patient reported frequent headaches, fatigue and intermittent claudication. She also presented features typical for TS: facial dysmorfism, webbing, low posterior hairline, proeminent, low set ears, broad chest, short stature (3,8 percentile for age), BMI of 20 and cardio-vascular modifications: grade III/6 systolic murmur in the left parasternal region, weak femural pulses upon palpation, and also significant differences between tensional values mesured on the right upper (156/94 mmHg; > 99th percentile for age and height) and lower extremities (104/69 mmHg). Electrocardiog- raphy revealed sinus rhythm with signs of left atrial and ventricular hypertrophy.

Upon echocardiographic evaluation coarctation of the aorta was confirmed with an associated malformation of the aortic valve, but ascending aorta diameters were found to be within normal range (Figure 1). A thoracic angio-CT scan was performed for the evaluation of the vascular anatomy (Figure 2). In this clinical setting, due to the simptomatology of the patient, the significant blood pressure differences and the inability to control the tensional values through standard medication, the indication for interventional therapy was established. Intraprocedurally, hemodynamic measurements revealed a significant narrowing at the level of the aortic isthmus, with a peak-to-peak gradient between the ascending and descending aorta of $22 \mathrm{mmHg}$ (Figure $3)$. Stenting was performed under general anesthesia with a $34 \mathrm{~mm}$ polytetrafluoroethylene-covered CP stent (NuMED, NY, USA) mounted on a $16 \mathrm{~mm} / 4.5$ cm BiB balloon (NuMED, NY, USA) at nominal pressure and under rapid pacing. Post-dilatation measurements revelead a gradient of $1 \mathrm{mmHg}$ (Figure 4).

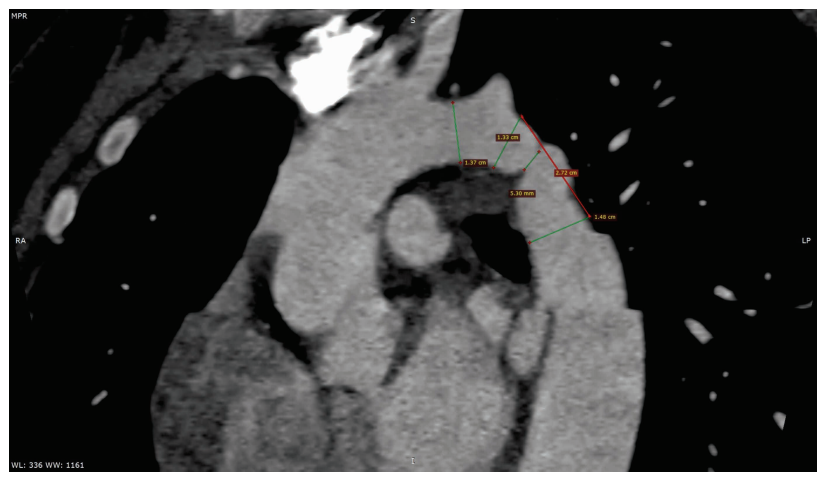

FIGURE 2. Thoracic angio CT-scan with aortic dimensions

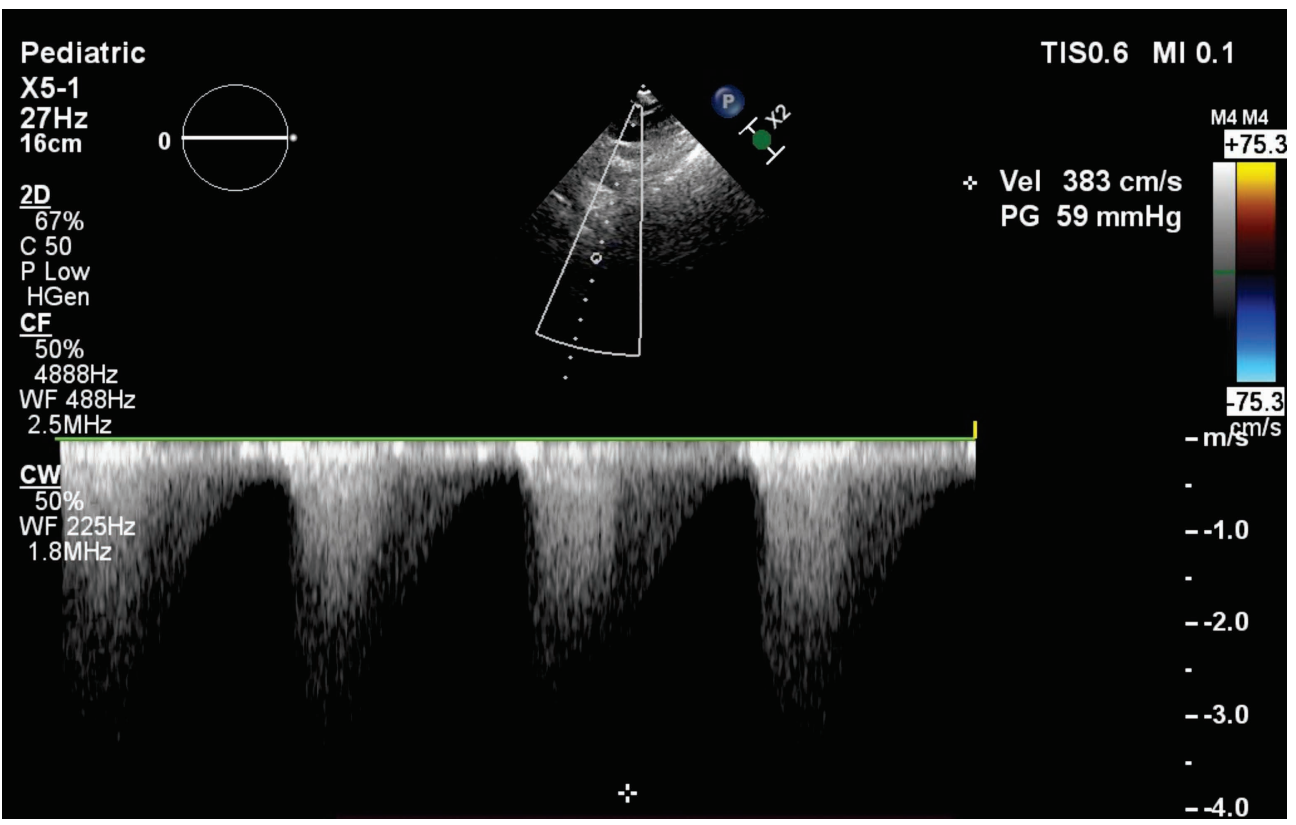

FIGURE 1. Suprasternal echocardiographic view showing a significant gradient at the aortic isthmus of $59 \mathrm{mmHg}$ 


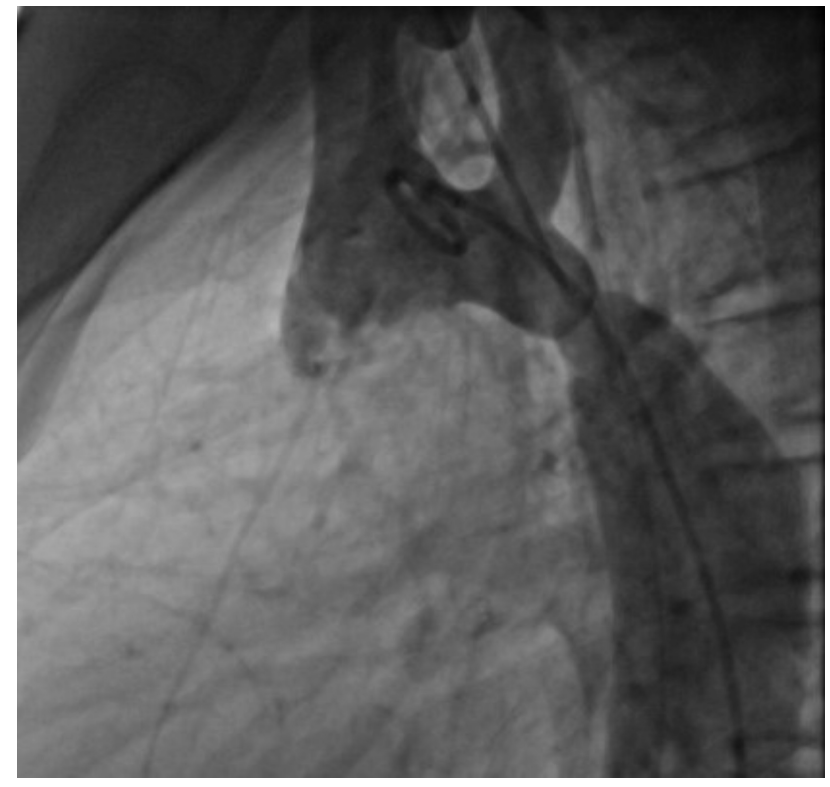

FIGURE 3. Angiographic image showing a significant narrowing at the aortic isthmus and post-stenotic dilatation

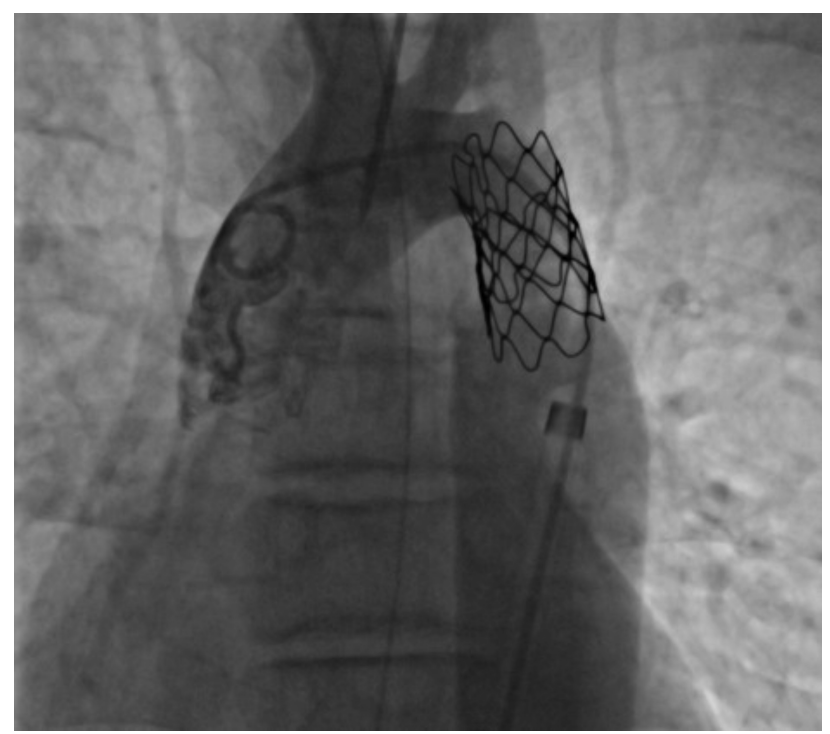

FIGURE 4. Antero-posterior view with the expanded stent at the site of the coarctation, being free from signs of dissection

The evolution of the patient was favorable, being free from procedure-related or post-procedural complications. Due to the maintainance of high blood pressural values, the patient was discharged with beta-blocking medication. Upon follow-up (at 3 months) echocardiography revelead no signs of dislocation, in stent stenosis, re-coarctation or dissection, the patient remained asymptomatic and blood pressure values remained in the normal range for age and height under beta-blocking medication.

\section{DISCUSSION}

Congenital heart disease can be present in up to $50 \%$ of TS patients, having a higher incidence in cases with 45,X monosomy, rather than in mosaics or other structural abnormalities [1,2].

In a large reprospective study, Yetman et al. found that congenital heart defects affect 56\% (321/569) of TS patients, from which 49\% (159/321) required a median of 1 (1-6) percutaneous or surgical cardiac interventions [5]. Regarding the spectrum of the defects, bicuspid aortic valve was found to be the most frequent, with a prevalence of $40 \%$, followed by coarctation of the aorta with $22 \%$ [5]. In our case, echocardiographic evaluation firstly raised the suspicion of a bicuspid aortic valve, diagnosis which was not sustained by the performed thoracic CT-scan. Thus, we wish to underline the importance of advanced imaging techniques in the diagnostic process of these patients.

From another perspective, diagnosis of coarctation of the aorta or bicuspid aortic valve in female patients may represent independent markers for TS, thus supporting the need for genetic evaluation and screening in this group $[2,4,6]$

Madriago et al. aimed to analyze the outcome of cardiac procedures in TS patients [7]. The group conducted a retrospective case-control study by analyzing data found in the Pediatric Cardiac Care Consortium database, from patients who underwent cardiac procedures in the 19 registered cardiac centers, between 1982-2006 [7]. Thus 398 TS patients where included [7]. Procedures involving left sided lesions were most frequent (95,2\%); from the surgical domain the most frequent was represented by repair of aortic coarctation (54,8\%) and amongst catheterization procedures balloon angioplasty for coarctation was performed most often $(9,4 \%)$ [7]. Regarding the outcome of procedures, overall mortality rates, hospital lenght of stay and number of admissions per patient where similar, whilst number of procedures per patient and age at the procedure where lower in the TS group, when compared to their non-TS counterparts [7].

In neonates and infants with coarctation, the procedure of choice remains surgical. However, in older children, transcatheter interventions have gained great importance. Stent placement is known to be an effective treatment, resulting in significant increase in aortic diameters, with subsequent decrease of systolic blood pressure and peak gradient [8]. Although reported complication rates in the general population are considered to be low [8], concerns in TS are heightened, due to associated aortopathy and data are somewhat conflicting.

Zanjani et al. reported their experience in stent implantation in ten TS patients with aortic coarcation with a median age of 12 years [9]. In the study group seven patients received bare metal stents, while covered stents were implanted in 3 patients [9]. No procedure-related complications where ob- 
served and after a median follow-up of 30,5 months, two patients developed aortic aneurysms at the coarctation site, all patients being free from restenosis [9]. The research group also conducted a literature review and concluded the following: when comparing the three available therapeutic options - surgery, balloon angioplasty and stenting- mortality and risk of aortic wall injury was highest in the surgical field and lowest in regard to balloon angioplasty [9].

A high adverse event rate was reported by van den Hoven in a multicenter, retrospective cohort study: despite being free from periprocedural complications, the short term follow-up (within the first 30 postprocedural days) of the included 19 patients (median age 16,9, 7 to 60 ) was marked by adverse events in 3/19 (15,8\%) of the cases (all adults, aged 47-48 years), including two dissections despite the usage of covered stents, one resulting in death [10]. In the third case direct casual relationship between the death of the patient and the stenting procedure could not be established as autopsy was denied [10]. It is also important to mention that both dissection cases had both high gradients and blood pressure values [10]. This finding can help underline the importance of adequate blood pressure control and assessment of the cardiovascular risk profile in TS patients. At long-term follow-up two additional, non-stent related events occured [10].

Although our case presentation holds valuable informations regarding the lack of periprocedural complications and favorable short-term evolution, our relatively short follow-up period represents a limitation. Thus we would like to underline the importance of a careful and systematic long-term evaluation of these patients, as determination of a longterm safety profile and complication rates of stenting in the pediatric age group remains an area of debate.

Pediatric data are scarce and limited to case reports, thus should be handled with care. Kataoka et al. reported the successful transcatheter management of native coarctation in 3 children with TS [11]. The procedures, including 2 stent placements and 1 balloon angioplasty, at a follow-up period of 3-4 years, where all free from complications [11]. On the other hand, authors have reported severe complications after percutaneous stenting of coarctation in an adolescent [12]. Fejzic et al. described the case of a 19 year old TS patient who suffered a fatal dissection of the descending aorta after she had undergone a two-staged implantation of a stent to relieve aortic coarctation [13].

Aortic dissection represents a severe, or even lethal complication in TS and may occur at smaller ascending aortic diameters in comparison to other aortopathies and at a median age of 29-35 years
[2,13]. Previosly published results show great variation regarding incidence $[13,14]$. Yetman et al. reported a dissection rate of $4.1 \%$ in an adult study group of 392 TS patients [15]. Patients in the pediatric age group with aortic dilatation and rapid progression of the aortic growth rate have also been reported to be vulnerable to dissection [16]. Hypertension, aortic dilatation, a bicuspid aortic valve, history of aortic coarctation, prior cardiac surgery, rural residence and the lack of ongoing cardiac care, all represent important risk factors $[13,15,17,18]$.

Lopez et al. have found TS to be an independent risk factor for aortic dilatation, suggesting an underlying aortopathy [19]. Additionally, TS associated vasculopathy has been described in the literature by increase in vascular stiffness and greater arterial medial thickness, and also cystic medial degeneration $[2,13,16]$. In this clinical setting of associated aortopathy, the implantation of a covered stent for the relief of coarctation is considered appropriate in these patients [11].

Given the related aortopathy and great risk for dissection, preventive measures were undertaken intraprocedurally such as covered stent placement and stent expansion at nominal pressures.

Systemic hypertension may affect $25-30 \%$ of children with TS and indicates risk for aortic root dilatation, with subsequent dissection and sudden death, thus justifying an agressive anti-hypertensive treatment protocol in these patients $[1,20]$. Encouragement of a healthy lifestyle and the aggressive management of obesity is primordial [2]. Medications such as beta-blockers, angiotensin receptor antagonists (both medications are effective in decreasing the aortic dilatation velocity), or angiotensin converting enzyme inhibitors, are warranted, but calcium inhibitors increase the risk of lymphoedema [1,2,21].

In this clinical setting, determination of the cardiovascular risk profile of these patients is of primordial importance, as both congenital and acquired heart diseases represent conditions of great risk for increased morbidity and mortality. Regular follow-ups should include blood pressure monitoring and echocardiographic evaluation of the progression of aortic stenosis, aortic regurgitation, aortic dilatation and risk assesment for dissection $[1,21]$. It is also important to underline that TS associated metabolic abnormalities, including dyslipidemia, diabetes, obesity and hyperuricemia, may lead to the exarcerbation of hypertension, coronary artery disease, myocardial infarction, or even stroke, thus warranting a multidisciplinary approach for these patients [2].

Early diagnosis of TS provides opportunities for treatment, including hormone and fertility optimi- 
zation, cardiovascular screening, and also treatment of cognitive and neurodevelopmental issues [4].

\section{CONCLUSIONS}

As a gold standard is not yet established for the treatment of TS-associated aortic coarctation, the present work would like to emphasize the role of minimally invasive interventional therapy and to

\section{REFERENCES}

1. Y Dulac, C Pienkowski, S Abadir et al. Cardiovascular abnormalities in Turner's syndrome: What prevention? Archives of Cardiovascular Disease. 2008; 101:485-490.

2. M Silberbach, JW Roos-Hesselink, NH Andersen et al, on behalf of the American Heart Association Council on Cardiovascular Disease in the Young; Council on Genomic and Precision Medicine; and Council on Peripheral Vascular Disease. Cardiovascular Health in Turner Syndrome: A Scientific Statement From the American Heart Association. Circ Genom Prec Med. 2018; 11: e000048.

3. R Rapaport. Hypofunction of the ovaries. In RE Behrman, RM Kliegman, Hal B Jenson (Eds.) Nelson Textbook of Pediatrics- Edition 17. Elsevier Philadelphia. 2004; 1931-1934.

4. A Eckhauser, ST South, L Meyers et al. Turner Syndrome in Girls Presenting with Coarcation of the Aorta. J Pediatr. 2015; 167: 1062-1066.

5. Yetman AT, Starr L, Sanmann J et al. Clinical and Echocardiographic Prevalence and Detection of Congenital and Acquired Cardiac Abnormalities in Girls and Women with the Turner Syndrome. Am J Cardiol. 2018; 122(2): 327-330.

6. Wong SC, Burgess T, Cheung M et al. The Prevalence of Turner Syndrome in Girls Presenting with Coarctation of the Aorta. J Pediatr. 2014; 164(2): 259-263.

7. Madriago E, Nguyen T, McFerson M et al. Frequency and Outcomes of Cardiac Operations and Catheter Interventions in Turner Syndrome. Am J Cardiol. 2012; 110: 580-585.

8. Hartman EMJ, Groenendijk IM, Heuvelman HM et al. The effectiveness of stenting of coarctation of the aorta: a systematic review. Eurolntervention. 2015; 11: 660-668.

9. Zanjani KS, Thanopoulos BD, Peirone A et al. Usefulness of Stenting in Aortic Coarctation in Patients With the Turner Syndrome. Am J Cardiol. 2010; 106: 1327-1331.

10. van den Hoven AT, Duijnhouwer AL, Eicken A, et al. Adverse Outcome of Coarctation Stenting in Patients with Turner Syndrome. Catheter Cardiovasc Interv. 2017;89(2):280-287. underline the need of further, larger scale studies and guidelines in this patient group. Given the related aortopathy, all preventive measures should be undertaken to avoid aortic dissection during stent implantation in this vulnerable population. Although the evolution of our patient was favorable, data found in the literature is somewhat contradictory and a close follow-up is indicated to help evaluate the risk of long-term complications.

\section{Conflict of interest: none declared Financial support: none declared}

11. Kataoka K, Ozawa A, Inage A, et al. Transcatheter Repair of Native Coarctation in Children with Turner Syndrome: Three Case Reports and Literature Review. Congenit Heart Dis. 2006;1:315-320.

12. Rato J, Martins D, Mendes IC, et al. A sudden turner of events. J Card Surg. 2019;34(5):363-366.

13. Fejzic Z, van Oort A. Fatal dissection of the descending aorta after implantation of a stent in a 19-year-old female with Turner's syndrome. Cardiol Young. 2005;15:529-531.

14. Thunstrom S, Krantz E, Thunstrom E, et al. Incidence of Aortic Dissection in Turner Syndrome- A 23 Year Prospective Cohort Study. Circulation. 2019;139:2802-2804.

15. Yetman AT, Bisselou KSM, Sanmann JN, et al. Vascular dissection in women with Turner syndrome. International Journal of Cardiology. 2021;325:127-131.

16. Pleskacova J, Rucklova K, Popelova J, et al. Aortic dissection and rupture in a 16-year-old girl with Turner syndrome following previous progression of aortic dilation. Eur J Pediatr. 2010;169:1283-1286.

17. Duijnhouwer A, Bons LR, Timmers HJLM, et al. Aortic dilatation and outcome in women with Turner syndrome. Heart. 2019; 105(9):693-700.

18. Carlson M, Airhart N, Lopez L, et al. Moderate aortic enlargement and bicuspid aortic valve are associated with aortic dissection in Turner syndrome: report of the international Turner syndrome aortic dissection registry. Circulation. 2012;126(18):2220-2226.

19. Lopez L, Arheart KL, Colan SD, et al. Turner Syndrome Is an Independent Risk Factor for Aortic Dilation in the Young. Pediatrics. 2008;121:e1622-e1627.

20. Allybocus ZA, Wang C, Shi H, Wu Q. Endocrinopathies and cardiopathies in patients with Turner syndrome. Climacteric. 2018 Dec;21(6):536-541.

21. Turtle EJ, Sule AA, Webb DJ, et al. Aortic dissection in children and adolescents with Turner syndrome: risk factors and management recommendations. Arch Dis Child. 2015;100(7):662-666. 